DOE/ER/40523--1

DE9 1007325

\author{
Progress Report \\ Period: March 1, 1990 to December 31, 1990
}

U.S. Department of Energy Contract No. DE-AS05-89ER40523

\title{
EXPERIMENTAL STUDY OF HEAVY FLAVOR PHYSICS AND SSC RESEARCH AND DEVELOPMENT at The University of Mississippi
}

\author{
Principal Investigator \\ James J. Reidy \\ Chair and Professor of Physics \\ Physics Department \\ University, MS 38677
}

\section{DISCLAIMER}

This report was prepared as an account of work sponsored by an agency of the United States Government. Neither the United States Government nor any agency thereof, nor any of their employees, makes any warranty, express or implied, or assumes any legal liability or responsibility for the accuracy, completeness, or usefulness of any information, apparatus, product, or process disclosed, or represents that its use would not infringe privately owned rights. Reference herein to any specific commercial product, process, or service by trade name, trademark, manufacturer, or otherwise does not necessarily constitute or imply its endorsement, recommendation, or favoring by the United States Government or any agency thereof. The views and opinions of authors expressed herein do not necessarily state or reflect those of the United States Government or any agency thereof. 
Table of Contents

Fuge

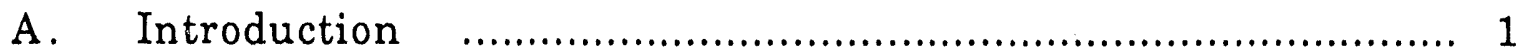

B. Progress in the Experimental High Energy Physics Program

During the Period $3 / 1 / 90$ to $12 / 31 / 90$.................................. 2

1.E691 ….......................................................... 2

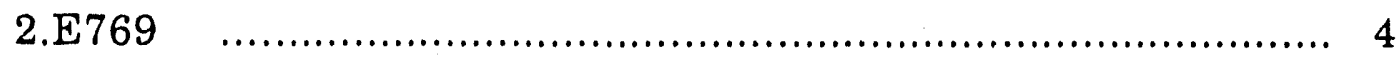

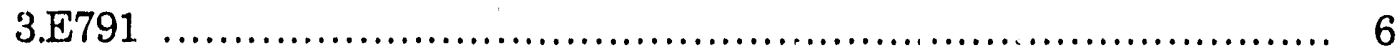

C. Progress in the SSC Research and Development Program ......... 12

1.CALOR/GEANT Integration ....................................... 13

2.The Calorimeter Simulation Effort ................................. 13

3.Scintillating Plate/Liquid Argon Calorimeter Simulation ......... 18

4.Liquid Scintillator R\&D .......................................... 19

5.Funding by the TNRLC .......................................... 20

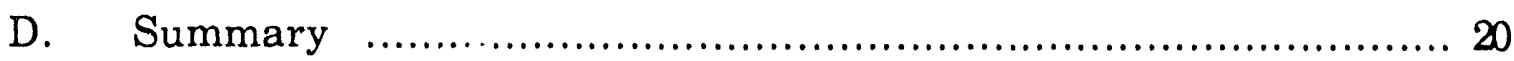

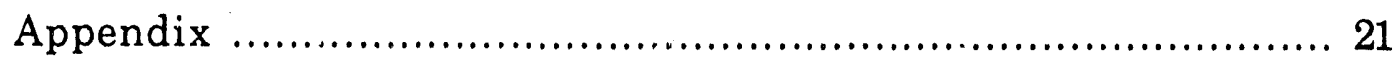

*1.Publications

2.Changes made to adapt EGSE for use on the Silicon Graphics

3 Changes made to adapt PEGS for use on the Silicon Graphics

4.Progress Report for the Scintillating Plate Calorimeter Subsystem

5.Progress Report for the Liquid Argon Subsystem

* copigraghtad papus 


\section{A.Introduction}

The University of Mississippi High Energy Physics (UMiss HEP) Group is partially supported by the U.S. Department of Energy contract no. DE-AS0589ER40523. It presently consists of four faculty, one postdoctoral associate, and five students with assistance from the physics department's electronics engineer, computer systems manager and administrative coordinator. Two of the students, Mr. Ali Rafatian and Ms. Danying Yi, are presently in residence at Fermilab. The UMiss HEP group has also established a cooperative program with the Memphis State University Physics Department whereby those students at Memphis State who are interested in high energy physics can work with our group and later transfer to the University of Mississippi as Ph.D. candidates in high energy physics. One student from Memphis State Univer iity, Mr. John Rose, was in residence at Fermilab and analyzed data from E691 as part of his Masters thesis. His work was co-directed by Dr. Cremaldi from the UMiss HEP group and Dr. Wayne Jones of Memphis State University. Two other students from Memphis State University, Mr. John Turner and Mr. John Furgeson, were supported by the UMiss HEP program and participated in E791 this past summer. Dr. Fred Lott, an assistant professor at Jackson State University (an HBCU) joined our group on E791 this summer as part of a cooperative research program we are establishing with them. In addition, three undergraduate students made significant contritutions to the E791 and SSC R\&D programs.

The HEP group is involved in three Fermilab experiments, E691, E769, and E791. In addition, the group has an ongoing SSC R\&D project dealing with the intergration of CALOR into GEANT and is participant on two SSC R\&D subsystem projects and co-spokesman on a fourth. The major contributions of the UMiss HEP group in these SSC efforts lie mainly in the areas of detector development and simulation and fast data acquisition systems. 


\section{B. Progress in the Experimental High Energy Physics Program during the period $3 / 1 / 90$ to $12 / 31 / 90$}

1.E691(Photoproduction of Charm): UCSB, Carelton U., U. Cincinnati, CBPF, U. Colorado, FNAL, U. Mississippi, NRC Canada, Princeton, U. de Sao Paolo, U. Toronto, Yale.

E691was a high statistics charm photoproduction experiment run at FNAL in the summer of 1985. It has produced the largest set of charm data every recorded and reconstructed in a fixed target experiment. About 15-20 journal articles, mostly in Physical Review Letters have been published. Additional papers are forthcoming. We estimate 20-30 papers will result in all. Five graduate students have completed thesis on E691; five more are now finishing.

We have about 20 invited talks appearing at conference proceedings over the years. Our charm results are still being shown at conferences and continue to be well received. The E691 analyses will continue to for at least one more year. The experiment should be noted for its stability and reliability, timely reconstruction of data, and excellent analysis record.

Dr. Cremaldi has published two papers on charmed baryon production and decays. He nas published a very accurate $\Lambda_{c}$ lifetime measurement, "Measurement of the $\Lambda_{\mathrm{C}}+$ Lifetime", which firmly established the lifetime at about 2.2(15\%) psec. In 1989 he published in PRL a measurement of the $\Sigma_{c}{ }^{O}$

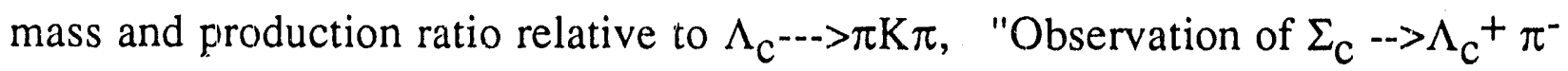
Decays". Two additional papers were published by Dr. M. Gibney at U. of

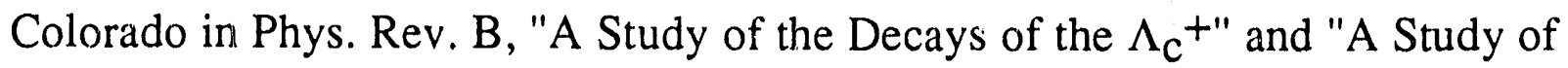
the Decays $\mathrm{D}-->\mathrm{K}^{0} \pi^{+}$and $\mathrm{D}-->\mathrm{K}^{\circ} \mathrm{K}^{+"}$. In all the Colorado/Mississippi group has been quite productive.

Dr. Cremaldi has supervised Master's Degree student, John Rose, from Memphis State University (Dr. Wayne Jones is John's advisor at MSU). He has completed a 
Master's Degree on $\psi^{\prime}-->\psi \pi^{+} \pi^{-}$decays from the E691 data sample entitled, "A Study on the Photoproduction of Psi-Primed Mesons".

Papers published by the E691 collaboration during the progress report period include (copies are in the Appendix):

1. "A Study of $\mathrm{D}_{\mathrm{S}}{ }^{+}$and $\mathrm{D}^{+}$Decays Into Four-Body Final States Including $\eta \pi^{+}$ and $\omega \pi^{+}$, The Tagged Photon Spectrometer Collaboration, J.C. Anjos et al., Phys. Lett. B223 (2), 267 (1989).

2. "A Study of the Decays of the $\Lambda_{c}{ }^{+"}$, The Tagged Photon Spectrometer Collaboration, J.C. Anjos et al., Phys. Rev. D 41, 805 (1990).

3. "A Study of the Decays of $\mathrm{D}-->\mathrm{K}^{\circ} \pi^{+}$and $\mathrm{D}-->\mathrm{K}^{\circ} \mathrm{K}+$ ", The Tagged Photon Spectrometer Col'x zboration, J.C. Anjos et al., Phys. Rev. D 41, 2705 (1990).

4. "Photon Gluon Fusion Analysis of Charm Photoproduction", The Tagged Photon Spectrometer Collaboration, J.C.C. Anjos et al., Phys. Rev. Lett. 65, 2503 (1990).

5. "Measurement of the Form Factors in the Decay D+ -.-> $\mathrm{K}^{*} \mathrm{o} \mathrm{e}^{+} \mathrm{v}_{\mathrm{e}}$ ", The Tágged Photon Spectrometer Collaboration, J.C.C. Anjos et al., Phys. Rev. Lett. 65, 2630 (1990).

Papers slated for 1991 publication include:

1. "Some Cabibbo-Suppressed Decays of the Do Meson", The Tagged Photon Spectrometer Collaboration, J.C.C. Anjos et al., Submitted to Phys. Rev. Rapid Communications.

2. "Study of the $\mathrm{D}_{\mathrm{S}}{ }^{+}-->\phi \mathrm{e}^{+} \mathrm{v}_{\mathrm{e}}$ and the Absolute $\left.\mathrm{D}_{\mathrm{S}}-\cdots\right\rangle \pi^{+}$Branching Fraction", The Tagged Photon Spectrometer Collaboration, J.C. Anjos et al., submitted to Phys. Rev. Lett. 
3. "A Study of the Deca: $D_{\mathrm{S}}{ }^{+} . .->\eta^{\prime} \pi^{+"}$, The Tagged Photon Spectrometer Collaboration, J. C.C. anjos et al., submitted to Phys. Rev. D Rapid Communications.

4. "Measurement of the Decays Do.$-->\pi^{+} \pi^{-}$and $D^{\circ}->K^{+} K^{-}$, The Tagged Photon Spectrometer Collaboration, J.C.C. Anjos et al., in preparation.

5. "Some Cabibbo-Suppressed Decays of the Do Meson", The Tagged Photon Spectrometer Collaboration, J.C.C. Anjos et al., in preparation.

6. "Measurement of $\Lambda_{c}{ }^{+. .->} \pi K^{*}$ o Decays", The Tagged Photon Spectrometer Collaboration, J.C.C. Anjos et al., in preparation.

2.E769(Hadroproduction of Charm): CBPF, FNAL, U. Toronto, U. Miss., Northeastern, Tufts, U. Wisconsin, Yale.

E769 was a charm hadroproduction experiment run at the Tagged Photon Spectrometer in the fall and winter of $1986 / 87$. The primary goals were to study the A-dependence and beam flavor dependence of producing charm particles in nuclear targets as well as production issues $\left(\mathrm{xF}_{\mathrm{F}}\right.$ ant $\left.\mathrm{P}_{\mathrm{t}}\right)$ in general. About 500 million events were recorded. It is the highest statistics charm hadroproduction experiment ever run at FNAL producing about 1/3-1/2 the charm sample of E691. This 3-4 thousand event charm sample is the largest ever accumulated by a fixed target hadroproduction experiment anywhere.

Almost all of E769's data has now been extracted and reconstructed from some 10,000 nine track tapes. A sub-sample of events has now been made based on the reconstruction of multiple vertices as seen in our silicon micro-vertex detector. Results on charm hadroproduction were presented at the XXVth International Conference on High Energy Physics in Singapore last August. A paper on A 
dependence will be submitted for publication in the near future. Ali Rafatian, who is working toward a Ph.D. at the University of Mississippi, is working on E769 analysis at Fermilab.

Dr. Don Summers and Dr. Chris Stoughton (Fermilab) put together a system of Silicon Graphics computers, which has been crucial to the successful analysis of E769. This system is based on RISC CPU's and UNIX and consists of four Silicon Graphics 4D/240 compute servers, one belonging to the Fermilab Physics Department and three devoted to E769 under the auspices of the Fermilab Computing Division. Each 4D/240 has four 25MHz MIPS R3000 RISC CPUs. Events are distributed from am $8 \mathrm{~mm}$ Exabyte tape drive to the $16 \mathrm{CPUs}$ using software developed by the Fermilab Advanced Computing Project (ACP). This farm of UNIX/RISC CPUs now accounts for one-quarter of the computing cycles available at Fermilab and is equal to over 250 VAX 11/780s. This system was used to reconstruct a large fraction of the E769 data sample. All the reconstructed tapes were then passed though this system to generate a sub-sample of events with secondary vertices. Reconstruction of E769's 500 million events was completed in the summer of 1990, a bit less than one year after all the computers necessary to analyze the experiment were brought on line.

Dr. Lucien Cremaldi has worked on the reconstruction of the Cerenkov counters for E769 and Dr. Don Summers has worked on the recontruction of the electromagnetic calorimeter.

\section{References}

D.J. Summers et al., Charm Hadroproduction at Fermilab E769, Proc. XXIIIrd Rencontre de Moriond, Current Issues in Hadron Physics, Les Arcs, France (1988) 451.

D. Errede et al., Hadroproduction of Charm at Fermilab E769, Proceedings of the XXVth International Conference on High Energy Physics (Singapore) 2-8 August 1990 (see Appendix). 
3.E791(Hadroprodoction of Charm): CBPF, FNAL, IIT, Univ. of Mississippi, Princeton, Ohio State Univ., Tel-Aviv Univ., Tufts Univ.,UFRJ, U. of Wisconsin, Yale.

E791 is a very high statistics ciarm hadroproduction experiment operating at a beam momentum of $500 \mathrm{GeV}$. Its goal is to record a data sample with over 100 $\mathrm{K}$ reconstructed charm particles. This experiment is based on the past successes of E691 and E769 at the Tagged Photon Spectrometer. With the implementation of a very fast data aquisitian system $(10 \mathrm{MB} / \mathrm{sec})$, fast front-end digitizers, compression, and recording of data about 4000 evts/sec can be recorded. During the test run in the summer of 1990 the upgrade of the experiment was completed and about one billion events were recorded in a three week period. Each billion events will result in about $10 \mathrm{~K}$ reconstructed charm particles. This experiment will allow the usual high statistics study of meson production, lifetimes, and spectroscopy, and also a first look at a range of rare decays, charm-strange meson and charmed baryon spectroscopy. Possibly a few 10's of B-mesons will be fully reconstructed.

\section{E791 Data Acquisition}

The E791 DA system collects data during a spill at the rate of $25 \mathrm{MB} / \mathrm{s}$. Data is stored in 8 large spill FIFOs. This allows building, compressing, and writing data to tape continuously. A wall of $428 \mathrm{~mm}$. Exabyte tape drives accepts dita at the rate of $10 \mathrm{MB} / \mathrm{s}$. Readout deadtime is typically 35 microseconds. This may improve. The front ends consist of LeCroy FERA ADCs, Phillips TDCs, LeCroy PCOS III latches, Nanomaker SMD latches, and a few CAMAC scalars and latches. The readout controllers include the Fermilab FASTBUS Smart Crate Controller, the Fermilab CAMAC Smart Crate Controller, Princeton SMD Scanners, and the Mississippi FERA/PCOS Controller. The UMiss Controller (UMC) was designed and built by Dan Kleinert of the University of Mississippi. See Figure 1. Steve Bracker, of the University of Toronto, led the E791 DA effort. Don Summers worked on the DA design and ferreted out many of the 


\section{The University of Mississippi Controller (UMC)}

The UMC is used to read out two of the E791 front end systems. Both use 16-bit ECL Lius to output data at the rate of one 16bit word every $100 \mathrm{~ns}$. The first system is the LeCroy PCOS III. It sets latch bits when Proportional Wire Chamber hits are detected. The second system is the LeCroy FERA ADC. It digitizes pulse heights from the E791 Cerenkov counters, electromagnetic, and hadronic calorimeters, ET trigger, and Transition Radiation Detector.

The UMC packs 16-bit words into 32-bit words and calculates a leading word count. A pair of UMCs is connected to a large spill FIFO. A third UMC shares a second large spill FIFO with a Smart Crate Controller. A token is passed to allow sequential readout on each chain. Once ds ta enters the small event FIFO on each UMC, the experiment is alive again. The readout time is about 30 microseconds The UMCs were designed and built by Dan Kleinert.

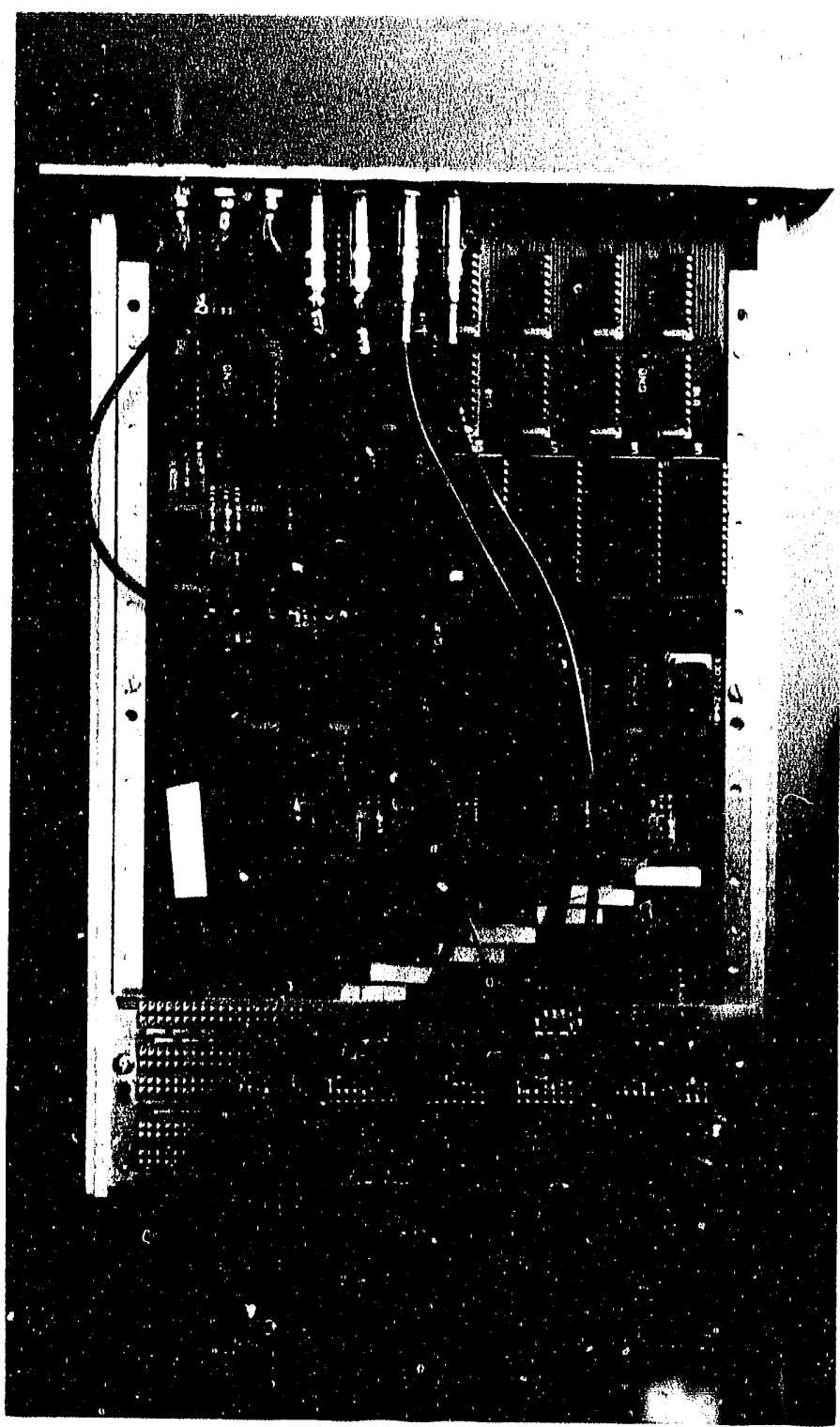

Figure 1 
parts which make up the DA, including the VDAS FIFO system, affordable DRAMs, VME crates, power supplies, Ciprico tape controllers, and $8 \mathrm{~mm}$ tape drives.

\section{Cerenkov Detectors}

Two large multi-cell Cerenkov detectors provide particle identification at the Tagged Photon Spectrometer (TPL). All physics done at TPL has depended in varying degrees on this system. No large upgrades have been made since E691, summer, 1984. The Cerenkov system has operated in a stable and efficient fashion since then under the supervision of Dr. Cremaldi.

Drs. Bolen and Cremaldi and students Eric Aitala, Trey Dillinger, Tim Rich, and John Rose successfully brought the E791 spectrometer Cerenkov counters up for the spring-summer 1990 run. Rose worked on the project until May 90 when he spent full time on his master's thesis. Aitala and Rich made important contributions in the summer tr keeping this system running. None of these students will be available for the upcoming run and as yet there are no Mississippi students or postdocs dedicated to the project for the 1991 run. Consequently,Dr. Cremaldi will have to assume responsibility for the system during the 1991 run.

The Cerenkov gas monitoring system for the 1990 run was not fully implemented. This was partially due to lack of funds and manpower. We relied on a few mass spectrometer samples evaluated at Fermilab and taken on a weekly basis to judge whether the gas mixtures in the Cerenkov counters were stable. This weekly monitoring seems adequate.

The FERA ADC readout of the cerenkov channels has worked well. The automatic pedestal subtraction gives stable results. It appears most calibration constants can be extracted from the data. Dr. Cremaldi and undergraduate student $T$. Hail have recently extracted su: ne of the calibration constants for the summer run. After the particle tracking results are available, a considerable software effort is needed to complete the Cerenkov calibration. Without 
adequate home computing or efficient links to Fermilab our active participation in software development is stymied. Collaborators at Tel-Aviv University have expressed interest in helping with software and calibration projects. Dr. Cremaldi is working with a student Sharon Bech from Tel-Aviv on Carenkov calibration and reconstruction.

\section{Muon Detection at TPL}

A major shortcoming of the E791 spectrometer at TPL is the muon detection. E691 has observed both $\psi \rightarrow->\mu \mu$ and D-->X $\mu \nu$ decays. The semimuonic signal is not competitive with the D-->Xev decays because of an added level of reconstruction background. This background is partially caused from the unavoidable $\pi$ and $\mathrm{K}$ decays in the spectometer, and reconstruction accidentals due to misassociated hadron track overlaps in the simple single layered TPL muon system. We have determined that even a mociest addition to the existing system will reduce backgrounds by a factor of ten. We have recommended and are implementing the addition of a second layer of scintillator paddles spaced behind the existing system. Although no funding was made available (we asked for $\$ 10 \mathrm{~K}$ in our 1989 proposal ) Dr. Summers, Dr. Cremaldi , and undergraduate Breese Quinn, a summer student from Mississippi State University, have resurrected some old scintillator found at FNAL and erected a new 18 paddle wall to enhance the E791 muon detection. The paddles were constructed under UMiss guidance at the FNAL scintillator shop headed by Ken Gray.

An additional 16 paddles of higher quality scintillator have been purchased by UMiss at a cost of about $\$ 10 \mathrm{~K}$. These paddles will be prepared for use at UMiss and are to be installed during the 1991 run of E791. We would have much preferred to do this work during the fall of 1990 at UMiss but were delayed due to lack of funds. We will rely on equipment from FNAL to test the paddles. A graduate student Danying $\mathrm{Yi}$ will $\mathrm{b}$ ? assigned to oversee all the muon systems for the 1991 run of E791. 


\section{PWC System}

The TPL PWC systems were commissioned by senior graduate student Ali Rafatian. Dr. Cremaldi guided this work through the 1990 run. This system is composed of eight beamline PWCs for beam particle tracking and two downstream Y-view planes mainly used for pattern recognition. There are abort 1000 channels in all.

The PWC hits are read into the DA through the UMMiss controller and the PCOS system. Kleinert and Rafatian have debugged this system and all known errors can be attributed to Lecroy Corp. We believe the UMiss controller is one of the sole devices which can strobe the PCOS system at high speed into a buffer memory. Rafatian has written online monitoring routines for the PWC system and has taken responsibility for this system during E791 running.

The beamline PWC system worked well during the 1990 run. These chambers are used in conjunction with the beamline silicon detectors provided by Princeton. Early analysis puts our total beam track finding efficiency near $98 \%$ for a snapshot of runs. Beam tracking will be used in our vertex reconstruction, especially in the implementation of a fast filter; thus the system has major importance.

The 500 channels of downstream PWCs were also functioning well during the summer run. Estimates of the efficiencies have not been made but some optimization seems necessary. Cremaldi and Rafatian will continue to work on the PWCs for the 1991 run.

\section{E791 Target}

Don Summers and Rick Milburn of Tufts built a new target for E791. It consists of one "high Z" platinum foil followed by four "low Z" diamond foils. 9mm decay volumes are provided between the foils. This will minimize background from secondary interactions. The geometry of the SMD system plus the desire for a $3 \%$ interaction length target dictated the choice of materials. 


\section{$\underline{\text { R3000 Developement }}$}

Dr. Summers and Memphis State University student John Turner worked with a "computer on a board" made by Integrated Device Technology. It was purchased with UMiss funds ( $\$ 1 \mathrm{~K}$ ) in the summer of 1990 for R\&D purposes. The board, IDTRS382, has a 16MHz MIPS R'30uU CPU and a R3010 FPU. It is one of a number of options we are exploring for offline reconstruction of E791. They compiled $C$ programs on a MIPS M/120 host computer and then downloaded and executed them on the IDT board. The board has r.urrently been loaned to CDF. It is bei"g used to test designs for the readout of FASTBUS crates in the new upgraded CDF data acquisition sytstem.

\section{Summer Students}

The UMiss HEP group had several student sunirner research assistants who worked on E791. Some of their contributions are described below,

Breese Quinn, an undergraduc te physics malor at Mississippi State Univerity, built a large fraction of a secrsnd muon wall for the detection of semi-leptonic decays of charm into muons. This second layer of scintillator paddles is orthogonal to those in the old wall. The purpose is to allow a muon hit to be matchec to the correct drift chamber track. He also devised and built a series of test units for checking connectors attached to 10,24, 50, and 64 conducter ribbon cables. He also took shifts.

John Turner, who is in the M.S. in Physics program at Memphis State University, worked with a "computer on a board" made by Integrated Device Technology. This is described above. John compiled C programs on a MIPS M/120 host. computer and then downloaded and executed them on the IDT board.

Eric Aitala, an M.S. student at the University of Mississippi, brought up the E791 Low Voltage Monitoring System. Almost 200 voltages are monitored including those from Data Acquisition, Silicon Microstrips, and Drift Chamber power supplies. High Voltages from the Drift Chambers, PWCs, and Cerenkov 
Counters are divided and read out as well. Six LeCroy CAMAC modules, each capable of scanning 32 channels, are used. Eric adapted an E769 FOR TRAN program to read out these CAMAC modules and display warning messages and worked with a new wiring scheme to connect power supplies to CAMAC. He also took shifts.

Tim Rich, a Ph.D. student at the University of Mississippi, wrote a Physics Analysis Workstation (PAW) computer program !sing KUMA.C to display and print a standard set of plots showing the performance of the calorimters and Cerenkoy counters. He also learned how to run the experiment and took shifts.

Trey Dillenger, an undergraduate in physics at the University of Mississippi, rebuilt the bucking coil system for the E791 Nitrogen Cerenkov counter. A more robnust power supply and wiring were installed. The magnetic fields at the faces of phototubes was checked with a Hall probe. Currents were adjusted to minimize the magnetic fields.

\section{$\underline{\text { References }}$}

D.J. Summers, Data Acquisition and Filtering, Physics at Fermilab in the 1990's, Breckenridge, Colorado (15--24 August 1989) 463.

\section{Progress in the SSC Research and Development Program during the period $3 / 1 / 90$ to $12 / 31 / 90$}

Currently our major efforts deal mainly with detector simulation calculations for the scintillating plate $R \& D$ program, the $L^{*}$ detector and the SDC detector. With Dr. Brent Moore joining our group in May, 1990, as a postdoctoral research associate this work has been carried out principally by Cremaldi, Moore and Reidy with assistance from an undergraduate student, Jaiping Zhang. Our output has been less than expected due to problems with our IBM-3084 out a CRAY XMP has just been installed on our campus and we are porting programs 
from LLNL which wil. enable us to use it in our detector simulation efforts. In addition, we are in the process of purchasing nearly 300 MIP's of Silicon Graphics computing using TNRLC and University funds. The University has installed a fiber optic connection from the HEP computing facility to the campus computer center so we now have the capability of high speed transfer via INTERNET.

\section{CALOR/GEANT Integration: Development of UNIX-based Code}

Dr. Moore, has been working on simulation related problems since last May. Although this position was primarily for a postdoc to work on our experiment at Fermilab (E791) $30 \%$ of his salary was from SSC generic R\&D funds. This was used as rationale for having him spend most of his time on SSC R\&D. Besides working on the scintillating plate $R \& D$ subsystem and $L^{*}$ calorimeter calculations Dr. Moore has spent considerable time converting CALOR to run on a UNIXbased system (Silicon Graphics). The CALOR/GEANT integration has been a major goal of the UMiss SSC R\&D effort. The procedure for obtaining this integration has changed from the original proposal primarily due to the rapid advances in UNIX-based microprocessor systems. The SSCL will have several thousand MIPs of UNIX-based microprocessors as their primary computing system, so we have decided that our greatest contribution to the program would be to install CALOR on a UNIX-based machine and then perform the integration in that environment. This would mean that both CALOR and the CALOR/GEANT integration package would be installed at the SSCL and thereby become available to the entire community. A number of technical problems have arisen in importing the CALOR program to our Silicon Graphics.

\section{The Calorimeter Simulation Effort}

The calorimeter simulation effort is based primarily on the CALOR89 code which has been developed at Oak Ridge National Laboratory. CALOR89 is a Monte Carlo simulation package designed to study the passage of radiation 
through calorimeters in elementary particle detectors. It consists of five major subroutines: HETC, EGS, MORSE, MOREGS and SPECT. HETC is the hadronic transport code, and the 'histories file' which it produces tells everything that happens to every particle that is created in the hadronic cascades. The neutrons that are created in this shower are analyzed by MORSE. The electromagnetic particles are analyzed by EGS, and the electromagnetic particles created by cascading neutrons are analyzed by MOREGS. Both EGS and MOREGS are very similar to the electromagnetic shower analysis code, EGSE. EGSE is the program used to measure the electromagnetic properties of showers. One generally sends incident electrons, positrons, or photons into the calorimeter. It is possible to determine the nature of the resulting showers and to measure the response of the calorimeter to these sh wers. SPECT is a program which does analysis on all the other types of particles. The output of the analysis routines is put into a final program which allows computation of such quantities as the electromagnetic resolution, the hadronic resolution, and the calorimeter response ratio $(\mathrm{e} / \mathrm{h})$. Many more details concerning the properties of a given calorimeter design may be found using this set of programs.

The calorimeters which we at the University at Mississippi have been simulating thus far have all been 'slab' calorimeters. These calorimeters are essentially rectangular prisms which are uniform in the $\mathrm{X}$ - and $\mathrm{Y}$-dimensions, but which have some variation of material in the Z-direction. The widths (in the $\mathrm{X}$ - and Ydirections) which we have been using have almost always been 2 meters by 2 meters. We have usually run simulations of sampling calorimeters, which have slabs of absorbing material such as iron or lead followed by slabs of plastic scintillator. One slab of each constitutes a unit cell, and we use enough unit cells to provide for eight interaction lengths of absorber. The incident particles then propagate in the Z-direction from the front of calorimeter to the back through the alternating layers of absorber and scintillator.

Simulations have been run as well for calorimeters which include a block of barium fluoride crystal. Such a has the same width as the calorimeter as a whole, but extend in the $\mathrm{Z}$-direction for 50 centimeters. Barium fluoride is a 
scintillating material, and these blocks have excellent spatial and energy resolution capabilities for electromagnetic showers. It is also possible to use CALOR to simulate barrel calorimeters as well, such as might be use to surround the interaction region of a collider, but we have not yet run simulations for this type of calorimeter. Recently we ran some simulations for the scintillating plate collaboration in conjunction with the University of Tennessee, Oak Ridge National Laboratory and Argonne National Laboratory. Our part in this collaboration was to run simulations for $10 \mathrm{GeV}$ pions and electrons incident on iron/scintillator sampling calorimeters. During one set of runs the iron thickness as held constant as the scintillator thickness was varied, while during the other set of the runs the thickness of the scintillator was held constant and the thickness of the iron was varied. The hadronic and electronic resolutions and the $\mathrm{e} / \mathrm{h}$ ratio were then examined as a function of the ratio of absorber thickness to scintillator thickness. A table with the results is included(see section C.3). The e/h ratio remained essentially constant at about 1.2. The resolution for both incident hadrons and electrons seems to grow worse, with very large absorber thicknesses giving the worst resolution. This is expected, as the shower is poorly sampled with very thick absorber slabs. As a part of the scintillating plate collaboration, we also ran a cross-check using lead as the absorbing material. We found the hadronic resolution for pions at $10 \mathrm{GeV}$ to be $(9.8+/-0.7) \%$. The electronic resolution was $(5.4+/-0.3) \%$, and the $\mathrm{e} / \mathrm{h}$ ratio was $0.97+/-0.01$. We ran the simulation for the same lead/scintillator valorimeter with a 50 centimeter block of barium fluoride on the front. The electronic resolution improved drastically to $(0.17+/-0.02) \%$. Other numbers were not as good, however, as the hadronic resolution increased to $(13.2+/-1.0) \%$ and the e/h ratio of $1.48+/-0.02$. Another run using incident particles of $5 \mathrm{GeV}$ instead of $10 \mathrm{GeV}$ gave an electronic resolution of $(0.46+/-0.03) \%$, a hadronic resolution of $(19.8+/-1.7)$ $\%$, and an e/h ratio of $1.56+/-0.02$. One point to mention about the $10 \mathrm{GeV}$ run with the barium fluoride is that the electron and photon cutoff energies in EGSE had to be increased by a factor of 10 in order to complete the electronic shower simulation in a reasonable amount of time. Even then the program took two-anda-half days of real time. 
This brings up some problems with the computer that is currently being used to run these programs at the University of Mississippi. It is rare that one has such a long time in which to complete the execution of a program. Currently, we are running CALOR on an IBM 3084 mainframe computer owned by the university and operated by the computer center. This computer is shutdown on a daily basis every morning for scheduled maintenance. As the permanent disk space allotted our accounts is limited, this means we must establish a temporary working space on a virtual disk each morning. This include formatting the virtual disk, and, as many of the history files from HETC are quite large, this process can take ten to twenty minutes. It is also necessary to store the history files on tape, since they are much too large to keep on our permanent disk space. Thus, every time we wish to run an analysis program using one of the history files, we mist load it in from tape. This adds another fifteen to thirty minutes to the time it takes to prepare to run programs, depending on how quickly the computer operator decides to mount the tapes. Thus, it takes at least 25 to 50 minutes just to prepare the computer for running a program such as SPECT, MORSE, or EGS, not counting the time it takes to make necessary alterations to the code to account for material type and location.

Output files which are large must be saved to tape upon the completion of the programs generating them, which sometimes requires the inconvenience of getting up at 4:00 or 5:00 AM to save the files before the computer is shut down at 7:00 AM. Perhaps the most restrictive effect of the daily shutdowns is that, generally, programs have less than 23 hours of real time in which to run. Given the many people, campus-wide, who use the machine, this amount of time can be used up easily. Further, under no circumstances is it feasible to generate a history file so large that it can not be stored on one tape. This usually means that we can run no more than 250 events a time and often no more than 200 events. This is opposed to the 1000 events that would be more statistically meaningful. In addition, the IBM 3084 uses a VM/CMS operating system which is awkward to use and very inflexible, the computer often undergoes unexplained system crashes 
and unannounced shutdowns, and we have even lost access to read and write to our own tapes on a few occasions until we were able to notify the appropriate person.

For these reasons and because it would make the code much more useful to the community, we have been trying to bring up the CALOR package on a UNIXbased RISC workstation: a Silicon Graphics Personal Iris 4-D. This will allow us to run simulations for large numbers of events at a time with several hundred megabytes of permanent disk space, easy storage on $8 \mathrm{~mm}$ exabyte tapes that hold over 2 gigabytes each, and a system that can be expected to provide much more continuous running time than the IBM 3084 mainframe. The Silicon Graphics is also expected to be much faster. The aforementioned barium fluoride simulation that took two-and-a-half days to run on the IBM 3084 took two-and-a-half HOURS to run on the Silicon Graphics. When we succeed in bringing up the code on the Silicon Graphics, this will be of substantial benefit to several other research groups as well, as there are several people who would be interested in running CALOR on UNIX-based systems.

Already, EGSE has been made to run on the Silicon Graphics( see Appendix). In order to adapt EGSE to run on this machine, all block data routines had to be named, the way random numbers were generated had to be changed, and some assembly code functions t.lat appeared to be doing bitwise manipulations of floating point numbers were replaced with standard functions that do bitwise manipulations on integers. In addition, PEGS, which prepares the cross-section input file for all the EGS-type routines, had to be changed( see Appendix). Specifically, 'nml=' had to precede the names of all namelist that were read from input files, the delimiters for the namelists in these input files had to be changed from ampersands (\&) to dollar signs (\$). In addition, it seemed to help to eliminate extraneous spaces in lines after the end of namelists. An 'external F' statement was added to the beginning of function DCADRE. Also, some variables passed to this integration function, DCADRE, were declared REAL in this function but had been declared REAL*8 in the function calling it. This was causing REAL problems. 
The only other programs we expect to have any trouble getting to run are HETC, SPECT, and MORSE. Actually, SPECT compiled with no problems as soon as it was put on the Silicon Graphics, but we have no HETC history file to use as input with which to run it and see if it works. Considerable progress has been made already on getting HETC to run on the Silicon Graphics, but yet more is required. No work has yet been done on MORSE. We have been given money with which to buy more UNIX machines, and it will be very easy to keep them busy. A $10 \mathrm{GeV}$ event for a single incident particle is the kind of simulation we've been running so far, and this provide useful information, certainly. It is, however, a far cry from simulating all the particles that result from the collision of two protons with each $20 \mathrm{TeV}$ energy. In order for simulations to be of much use in helping to designing calorimeters for the SSC, they need to be carried out within the next two to three years.

There are simulations which have been run in the Mississippi high energy physics group besides those involving CALOR. Lucien Cremaldi and Don Summers have proposed a high luminosity detector for a collider which consists of an electromagnetic calorimeter very near the interaction region and several outlying muon chambers. This design is intended to look for decays of the Higgs particles into two Z's, one of which subsequently dacays into an electron-positron pair, and the other of which decays into a pair of oppositely charged muons. GEANT simulations, including lego plots, have been generated to gauge the ability of the electromagnetic calorimeter in this detector to detect and isolate electronic showers that might result from such an interaction.

\section{Scintillating Plate/Liquid Argon Calorimeter Simulation Calculations}

The University of Mississippi, the University of Tennessee and Oak Ridge National Laboratory have been spearheading the efforts to benchmark the CALOR89 code system against the ZEUS, D0 and HELIOS prototype calorimeter data(see Appendix). This work is continuing. Some of our results for the 
lead/scintillator geometries are given below in Table 1.

\author{
Table 1 \\ University of Mississippi Results. \\ Scintillating Plate Collaboration Runs
}

Iron/Scintillator runs were all 250 events at $10 \mathrm{GeV}$.

\begin{tabular}{llrrc} 
Iron & \multicolumn{1}{l}{ Scint } & Had. Res.(\%) & \multicolumn{1}{c}{ EM Res. $(\%)$} & $\mathrm{e} / \pi$ \\
$1.0 \mathrm{Xo}$ & $0.10 \mathrm{~cm}$ & $15.2+/-1.1$ & $7.6+/-0.5$ & $1.23+/-0.02$ \\
$1.0 \mathrm{Xo}$ & $0.25 \mathrm{~cm}$ & $16.4+/-1.2$ & $5.9+/-0.3$ & $1.29+/-0.02$ \\
$1.0 \mathrm{Xo}$ & $0.50 \mathrm{~cm}$ & $15.4+/-1.3$ & $4.5+/-0.2$ & $1.17+/-0.02$ \\
$1.0 \mathrm{Xo}$ & $1.00 \mathrm{~cm}$ & $12.5+/-0.9$ & $4.6+/-0.3$ & $1.19+/-0.01$ \\
& & & & \\
$0.5 \mathrm{Xo}$ & $0.25 \mathrm{~cm}$ & $12.4+/-0.8$ & $4.3+/-0.2$ & $1.21+/-0.01$ \\
$1.0 \mathrm{Xo}$ & $0.25 \mathrm{~cm}$ & $16.4+/-1.2$ & $5.9+/-0.3$ & $1.29+/-0.02$ \\
$2.0 \mathrm{Xo}$ & $0.25 \mathrm{~cm}$ & $20.2+/-1.7$ & $9.0+/-0.7$ & $1.20+/-0.02$ \\
$4.0 \mathrm{Xo}$ & $0.25 \mathrm{~cm}$ & $28.7+/-3.1$ & $16.5+/-2.1$ & $1.21+/-0.02$
\end{tabular}

Lead/Scintillator run was 200 events at $10 \mathrm{Gev}$.

Lead Scint Had. Res.(\%) EM Res.(\%) e/ $\pi$

$\begin{array}{lllll}1.0 \mathrm{Xo} & 0.25 \mathrm{~cm} \quad 9.8+/-0.7 & 5.4+/-0.3 \quad 0.97+/-0.01\end{array}$

In addition, the University of Mississippi has a sizable role in the beam test program of the scintillating plate SSC R\&D subsystem group. Dr. Reidy, a postdoc and a student will assist in the construction of the hanging file calorimeter and will participate in the beam tests.

\title{
4.Liquid Scintillator R\&D
}

The University of Mississippi is co-spokesman for SSC Proposal No. 247: 
"Liquid Scintillator Calorimetery". Our major responsibility is to coordinate the calculational effort and perform some measurements. This prop sal has been approved for funding by the SSCL and discussions are in progress dealing with the MOU.

\section{Funding by the Texas National Research Laboratory Commission(TNRLC)} The TNRLC has awarded the UMiss HEP group $\$ 200,000$ for the purchase of computing equipment and support of a research scientist to coordinate the calorimeter simulation activities. The equipment funds will be used to purchase a UNIX-based microprocessor farm of about 300 MIPS.

\section{Summary}

The University of Mississippi High Energy Physics group has made extensive cnntributions in the field of charm physics and SSC R\&D. We have made a major contribution to a highly successful first run on E791 at Fermilab where over one billion events have been recorded on tape. One student has analyzed data from E691 for his M.S thesis work and two students are currently analyzing data from E769 for their Ph.D. dissertations. Steady progress in calorimetry simulation for SSC type detector geometries has occurred. The group is playing an important role in three major subsystem proposals and in developing tools for use by the community. 
APPENDIX

copyengter papers (removed and cycled sepmatily.

21 
Changes made to Adapt EGSE for Use on the Silicon Graphics*

The two BLOCK DATA routines were named BLKDT1 and BLKDT2, respectively. The Silicon Graphics will ignore all but the first BLOCK DATA if they are not given different names. The method of selecting random numbers was changed. As previously set up, a two-element INTEGER array, IX, was equivalenced to a double-precision real number, DRN. The first element in the array was given a fixed value in all routines (Hex - 46,000,000). The second element was set equal to a number, IXX, saved in common block RANDOM. To generate the random numbers, IXX is multiplied by a very large number (663608941) and IX(2) is set equal to IXX. Since DRN is equivalenced to IX, its value is changed. This seems to be done is such a way as to always generate a random number between 0 and 1 . All the following statements were removed: INTEGER IX(2) EQUIVALENCE (IX(1), DRN) DATA IX(1) / Z 46000000 /. The statements: IXX $=$ IXX $* 663608941$ IX(2) $=$ IXX, were then replaced with the call to FLRAN: call FLRAN(IXX,DRN). FLRAN is the name originally given by CALOR to an assembly code random number generator. A substitute subroutine FLRAN was used on the Silicon Graphics which effectively calls the Silicon Graphics random number routine, RAN, given IXX and returns DRN and a new value for IXX. Some changes were made to subroutine JOM6. Some assembly code functions were called in JOM6 which ran on the IBM 3084 but would not run on the Silicon Graphics. These appeared to do bitwise AND, OR and COMPL (complement) operators on floating point numbers. These functions were replaced by AND/IAND, OR/IOR and NOT/JNOT, respectively, which perform bitwise operations on integers. The old arguments of these functions were floating point numbers which had been equivalenced to integer numbers. When the changes to the integer bitwise operators were made, their arguments were also changed to the equivalent integer quantities. This involved the introduction of another equivalence: REG was equivalenced to IREG, a new variable. As JOM6 exists in most if not all the other CALOR programs, this change may be helpful in getting the rest of CALOR to run. 
* In addition to the changes made to adapt the EGSE program itself for use on the Silicon Graphics, changes were required in the cross-section preparation program, PEGS. No changes were made to the prepartion program, EGSEPREP. 
Changes made to Adapt PEGS for Use on the Silicon Graphics

Namelists are read in on lines $73,80,154$ and 335. In order to do this on the Silicon Graphics, it is necessary to type ' $\mathrm{nml}=$ ' before the name of the namelist. Therefore, this phrase has been included in these read statements. Namelists are included in all the input files, which are units 5,8 and 9. In all these files, the ampersands $(\&)$ were replaced with dollar signs $(\$)$ to prevent the Silicon Graphics from having difficulty in subsequent read statements. This is crucial for the unit 5 file, but is not so important for the unit 8 and 9 files. Sometimes it is necessary to trucate the unit 5 file so there are no extraneous spaces at the ends of the lines. It was also necessary to add the statement 'external $F^{\prime}$ to the beginning of the real function DCADRE. Finally, the declaration of variables has been changed in two functions. In function $Q D$, a number of variables were originally declared REAL*8. These variables were then passed to the integration function DCADRE where they were declared REAL. This was causing REAL problems. The declarations of the variables in both functions were changed to make them compatible. All variables in QD we declared to be REAL, and the variables in DCADRE were left declared as REAL except AERR and RERR which were passed as double-precision constants by $\mathrm{QD}$. These were declared REAL*8. 

